\title{
Adaptive Clustering with Dynamic Cluster Radius for Wireless Sensor Network
}

\author{
Luqiao Zhang ${ }^{1,2}$, Qinxin Zhu ${ }^{2}$ and Juan Wang ${ }^{1}$ \\ ${ }^{1}$ School of Network Engineering, Chengdu University of Information Technoloy, \\ Chengdu, China \\ ${ }^{2}$ School of Computer Science and Engineering, University of Electronic Science and \\ Technology of China, Chengdu, China
}

zhanglq@cuit.edu.cn,qxzhu@uestc.edu.cn,wangjuan@cuit.edu.cn

\begin{abstract}
Topology control in Wireless Sensor Network (WSN) has recently been an area of intense investigation, especially the cluster-based topology control algorithms. In this study, we propose Adaptive Clustering with Dynamic Cluster Radius (ACDCR), a cluster-based topology control algorithm. This protocol proves to have several salient features, such as higher energy efficiency, and better QoS (Quality of Service). The design is accomplished by employing the hop-adaptive cluster radius, cluster head selection criteria with left energy threshold, and several other mechanisms. In ACDCR, only nodes with higher residual energy level than their neighbors are identified as cluster head candidates. Each cluster is assigned with a hop related radius for load balancing. In addition, nodes are allowed to setup direct links with the base station, rather than only their cluster heads. Simulation study shows that ACDCR outperforms HEED (Hybrid Energy-Efficient Distributed Clustering) in terms of network lifetime and QoS with fairly reasonable tradeoffs.
\end{abstract}

Keywords: wireless senor network; topology control; clustering; quality of service; adaptive cluster radius

\section{Introduction}

After decades of research, Wireless Sensor Network (WSN) [1-2] has already been put into practices in different areas, including battle field surveillance [3], wild animal protection [4], and volcano monitoring [5]. Although extensive work has been done in this area, some problems have yet to be addressed and investigated [6]. One of the problems is how to deal with the frequent changes of underlying network structure, and provide high quality service to the end user (Here high quality service refers to longer network lifetime and better coverage.).

Due to frequent node failures, unstable wireless channel, and other reasons, the underlying network structure of WSN is more volatile than those of other networks. Such problem can be alleviated by topology control [7], which has already become one of the most important supporting techniques for WSN. Moreover, topology control also helps balancing load, saving energy, and reducing interference [8].

According to the overall network architecture, topology control algorithms can be categorized into two different types, hierarchical [9] and non-hierarchical [10]. In this study, we concentrate on the hierarchical algorithms with emphasis on cluster-based algorithms in the context of routing environment, and propose a clustering algorithm called Adaptive Clustering with Dynamic Cluster Radius (ACDCR). 
The rest of the paper is organized as follows. First of all, the taxonomy of clustering algorithms and the most typical approaches of each category is given. Secondly, the network model, including assumptions and notations, and the clustering problem are addressed. And then the operation of ACDCR is presented. Following is the analysis and simulation of ACDCR. At last, the conclusion is made and possible future work is listed.

\section{Related Works}

Topology control methods can be classified into different categories by using different criteria. Below are some most common criteria, followed by a brief introduction of the most typical ones.

\section{$\checkmark \quad$ Methodology}

The protocol can be executed in either centralized or distributed manner. It is preferred that the algorithm can be executed for each sensor node separately, which is helpful in large scale scenarios. However, centralized algorithms can be found in some early studies, for example LEACH-C [11].

\section{$\checkmark$ Assumption}

Topology control approaches are based on very different assumptions. For example, sensors are required to equip the location-aware module [12]; Nodes are assumed to have long-haul communication capability [13]. The topology control problem is studied with the existence of mobile agents [14].

\section{$\checkmark \quad$ Design Goals}

Algorithms are developed to achieve different design goals by applying different mechanisms. The earlier work focuses on energy efficiency [12]. Later, more factors are taken into account, such as the percentage of cluster head, the scale of each cluster, and other cluster characteristics [15].

A multi-criteria clustering method considering the number of cluster members and the total cost for all cluster members to communicate with their cluster head is proposed in [16]. A coverage and connectivity guaranteed clustering approach is presented in [17]. Intermediate mode, which uses cluster members for message relay, is introduced to increase the robustness and efficiency of the network in [18]. Overlapping clusters are formed instead of nonoverlapping clusters for the consideration of fault-tolerance in [19]. More examples can be found in [9].

\section{$\checkmark \quad$ Inter-cluster and Intra-cluster Topology}

The inter-cluster communication, i.e. the communication between the base station and cluster heads, can be arranged into single-hop [12] or multi-hop manner. In most cases, nodes are not capable of long-distance communication, so that it is well accepted that a multi-hop link should be setup between the base station and cluster heads. Moreover, generally speaking, the multi-hop mode can be more energy-efficient than the single-hop mode.

In contrast to inter-cluster communication, to avoid adding too much complexity, the most common solution for intra-cluster communication is to let cluster members communicate with their cluster head directly. However, in DWECH (Distributed Weight-based Energy-efficient Hierarchical clustering protocol) [20], lower energy consumption is achieved by generating a multi-hop intra-cluster topology. 


\section{$\checkmark \quad$ Algorithm Complexity}

The complexity of clustering approaches is different in terms of convergence time. For example, among the above mentioned algorithms, [12] terminates after constant rounds, while [11] has a time complexity of $O(n)$.

There are still more attributes, such as the cluster count in the network is variable or fixed, can be used to classify clustering algorithms. However, due to space limitation, we will not explore them in detail here.

By summing up the favored features for clustering algorithm, we here propose a distributed algorithm with constant convergence time called ACDCR. In ACDCR, mechanisms, like adaptive cluster radius and lower bound of residual energy for cluster head, are introduced to balance the load among nodes and achieve better coverage quality.

\section{Preliminary}

\subsection{Network Model}

The study is carried out under following assumptions, although all of them are widely accepted ones, for completeness, we still include them here. Moreover, for ease of illustration, some abbreviations and notations will be introduced.

1) All nodes and the $B S$ (Base Station) are stationary once deployed.

2) Sensor nodes are driven by batteries, while the $B S$ has sustainable energy supply.

3) All nodes are homogeneous, in terms of initial battery level and maximum transmission range.

4) All nodes are assigned with a unique $I D$, for example $i$ for the $i$ th node.

5) All nodes can adjust its transmission range between 0 and its maximum transmission range according to the requirement.

6) The approximate distance between node $i$ and $j$, denoted as $\operatorname{dist}(i, j)$, can be estimated by RSSI (Received Signal Strength Index), and thus GPS equipment is not required.

\subsection{The Clustering Problem and Proposed Solution}

In cluster-based algorithms, nodes are arranged into clusters. There is a $\mathrm{CH}$ (Cluster Head) in each cluster, and other nodes are called $C M \mathrm{~s}$ (Cluster Members), where a directly link is setup between the $C H$ and each $C M$. Thereafter, in the allocated TDMA slots, $C M$ s can transmit the sensed data to their $\mathrm{CHs}$, where the data can be further processed and then relayed to the $B S$.

The information needed for cluster formation is acquired through the exchanged information among one-hop neighbors. By the localized neighboring information, nodes decide whether to be Tentative_CHs or $\mathrm{CMs}$, common criteria for such decision include residual energy, node degrees, and so forth. Then a bunch of Final_CHs, which will be in charge of intra-cluster and inter-cluster communication, are selected from Tentative_CHs, while the rest nodes will step back to be $C M$ s.

In ACDCR, CMs are further divided into the FLCMs (First Level Cluster Members) and the $S L C M$ s (Second Level Cluster Member). For FLCM, it has a direct link to its $C H$, while $S L C M$ communicates with $C H$ by using FLCM as intermediate. Moreover, clusters have different cluster radius called ClusterRange to balance energy consumption. Note that, we 
focus on the process of clustering, so existed methods for inter-cluster communication will be put into use directly.

\section{The ACDCR Algorithm}

\section{Step I. Hop Initialization}

With the help of the $B S$, every node initializes its hops to $B S$. The $B S$ broadcasts a beacon message, and every node can sets its hops to $B S$ according to the received beacon message. Additionally, every node relays the beacon message with the hop information updated. Besides the hop information, other global settings like Intra $a_{0}$ and Intra ${ }_{\max }$ can be spread simultaneously in this step. The Intra $a_{0}$ and Intra $a_{\max }$ here denote the lower bound and upper bound for ClusterRange, respectively.

Hop Initialization is treated as a separate step, because unlike other steps, it only needs to be executed once during the network lifetime.

\section{Step II. Neighbor Discovery}

$$
\begin{aligned}
& \text { Range }_{i}=\left(1+\alpha \times \frac{\left(\text { Hop }_{i}+1\right)}{\max \text { hop }}\right) \times \text { Intra }_{0} \\
& \text { ClusterRange }_{i}=\min \left(\text { Range }_{i}, \text { Intra }_{\max }\right)
\end{aligned}
$$

In this step, for each node $i$, it first computes its own ClusterRange $e_{i}$ by (1) and (2), which are designed as a countermeasure to the 'funneling effect' [21].

It is known that nodes closer to the $B S$ drain out their battery more quickly because of the 'funneling effect'. Furthermore, the 'funneling effect' does not only lead to unbalanced energy consumption, it also results in network disconnection. Based on such observation, it is reasonable to let nodes, which have farther distance to the $B S$, have a larger cluster radius.

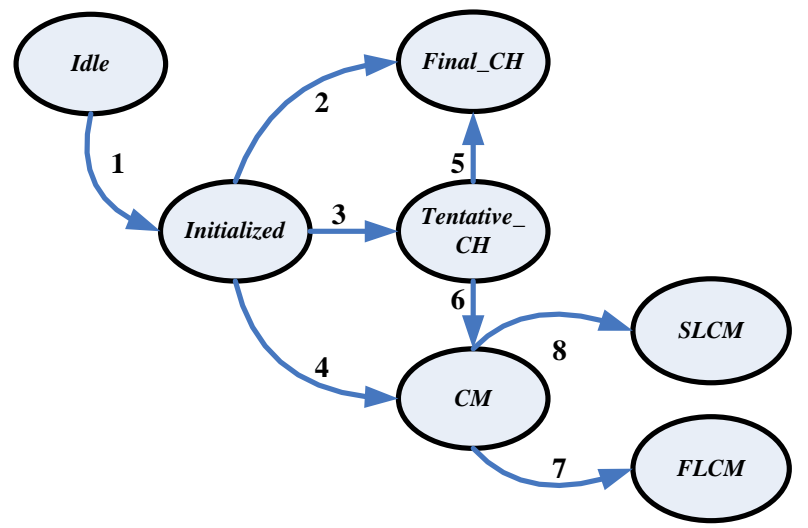

Figure 1. Node State Transition Diagram

A neighbor discovery message, including node $I D$, hop and residual energy, will be broadcasted by each node after the decision of cluster radius. And then every node within the distance of ClusterRange, will receive and reply for the message. It is worth noticing that nodes will reply the neighbor discovery message, regardless of its own ClusterRange. At the end of this step, nodes are considered Initialized (transition 1 in Fig. 1). 


\section{Step III. CH Election}

If node $i$ cannot find any neighbor inside its ClusterRange, it will become a $\mathrm{CH}$ directly (transition 2 in Figure 1). Otherwise it needs to decide whether to be a Tentative_CH or a $C M$ according to its residual energy level. As the average energy level of $i$ 's one-hop neighbors can be calculated, it will nominates itself as a Tentative_CH (transition 3 in Figure 1) if the condition described in (3) can be satisfied, else it chooses to be a $C M$ (transition 4 in Figure 1). Such setting will guarantee that nodes with rather low energy level will not be eligible candidates for $\mathrm{CH}$.

$$
E_{r}(i) \geq \operatorname{Avg}(i), \text { where } \operatorname{Avg}(i)=\frac{\sum_{j=1}^{\text {neighborcount }} E_{r}(j)}{\text { neighborcount }}
$$

For Tentative_CH $i$, it will determine to become a $\mathrm{CH}$ (transition 5 in Fig. 1) if CH_Prob(i), which can be computed by (4), reaches 1 . Otherwise, the $C H \_P r o b(i)$ will be doubled in next iteration. Moreover, the probability is not allowed to fall below $P_{\min }$ [15-16]. Such constraint is introduced to ensure that the $\mathrm{CH}$ election ends after constant iterations, which will be further illustrated in section IV.

$$
\mathrm{CH}_{-} \operatorname{Prob}(i)=\max \left(2^{\text {iteration }} \times\left(\frac{E_{r}(i)}{\operatorname{Avg}(i)}\right), 2^{\text {iteration }} \times P_{\min }\right)
$$

A $\mathrm{CH}$ announcement will be broadcasted by $\mathrm{CH} i$ within the radius of ClusterRange, if CH_Prob(i) reaches 1 . Nodes, which receive such announcement, will take actions according to their own states. For $\mathrm{CMs}$, they will include $i$ in their neighbor $\mathrm{CH}$ list for parent $\mathrm{CH}$ selection. As for Tentative_CHs, in addition, they will step back to $C M$ state (transition 6 in Fig. 1). For $\mathrm{CH}$ s, $\mathrm{CH} i$ will be stored in its $\mathrm{CH}$ list for inter-cluster link setup.

This step ends while there is no Tentative_CH node.

\section{Step IV. Cluster Joining}

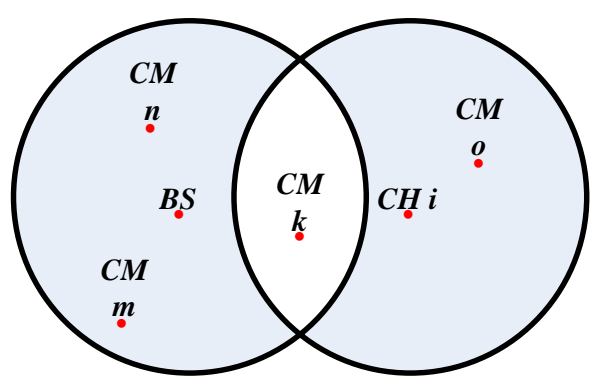

Figure 2. Cluster Joining Choice

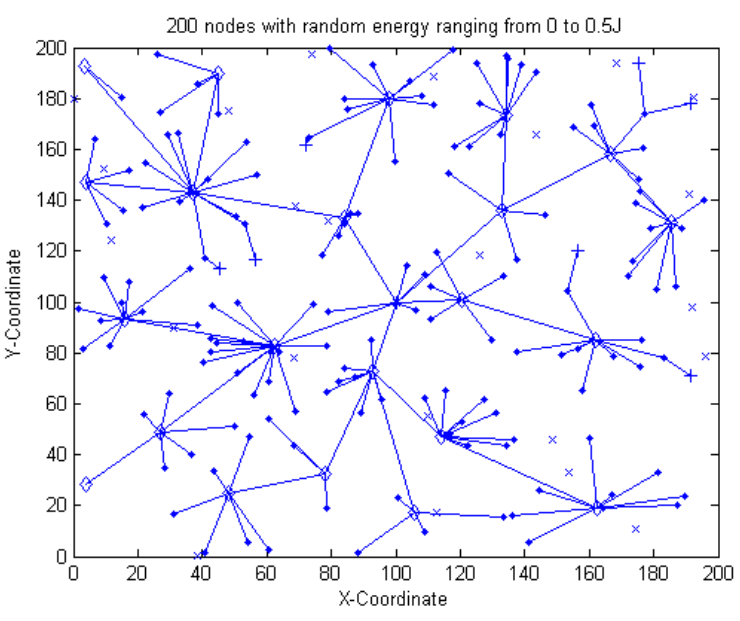

Figure 3. Topology Sample for ACDCR 
$C M$ s have to choose a cluster to join, i.e., choose a parent $C H$ (transition 7 in Figure 1). Here, the distance between a $C M$ and its potential parent $\mathrm{CHs}$ is used as a primary parameter for parent $\mathrm{CH}$ selection, and the potential parent $C H$ 's hop to $B S$ is used as the second parameter. And those nodes, which have a direct link to $C H$ s, are called FLCMs.

Moreover, there might be such situation as depicted in Figure 2. For $C M o$, which is covered only by $\mathrm{CH} i$, it will become the child of $\mathrm{CH} i$. For $\mathrm{CM} n$ and $\mathrm{CM} m$, which are covered by the $B S$ only, they will become the $B S$ 's children. As for $C M k$, the situation is a little complicated, it will become the child of the $B S$, if dist $(B S, k)$ is smaller than or equals to dist $(i, k)$. On the other hand, if dist $(B S, k)$ is bigger than dist $(i, k)$, it will choose $C H i$ to be its parent.

To round up, $C M$ s are allowed to setup direct link with the $B S$ in ACDCR. Such setting can further alleviate the 'funneling effect', because a message can be delivered to the $B S$ with less intermediate nodes.

One last question is how to deal with the 'orphaned' nodes, which are neither covered by any $C H$ nor the $B S$. For such node, it will become a child of the nearest FLCM (transition 8 in Figure 1), i.e. the SLCM.

\section{Step V. Backbone Network Setup}

This step deals with the problem of inter-cluster communication by setup a backbone network among $\mathrm{CHs}$. The main purpose of this step is to make sure that messages can be properly delivered to the $B S$, i.e., the network connectivity. So $\mathrm{CH} i$ selects the nearest $\mathrm{CH} j$ with a smaller hop than itself to be its parent $\mathrm{CH}$. In addition, the research result about how to preserve the network connectivity in [17] is applied here directly.

\section{Step VI. Data Collection}

\section{Table 1. Pseudo code of ACDCR}

\begin{tabular}{|c|c|}
\hline Step I. Initialization & END WHILE \\
\hline The $B S$ broadcasts a beacon message, and the & Step IV. Cluster Joining \\
\hline message is relayed with the hop information updated & FOR each $C M i$ \\
\hline by nodes. & $\mathrm{IF} \operatorname{dist}(i, B S) \leq \operatorname{dist}(i$, any $C H)$ \\
\hline Step II. Neighbor Discovery & direct link to the $B S$ \\
\hline FOR each pair of node $i$ and nod $j$, where dist $(i, j) \leq$ & ELSE \\
\hline ClusterRange $_{i}$ & IF $i$ is covered by any $\mathrm{CH}$ \\
\hline$i, j$ exchange $h o p$ and $E_{r}$ information & $F L C M \leftarrow i$ \\
\hline Step III. CH Election & ELSE \\
\hline FOR each node $i$, where $E_{r}(i) \geq A v g(i)$ & $S L C M \leftarrow i$ \\
\hline Tentative_CH $\leftarrow i$ & Step V. Backbone Network Setup \\
\hline WHILE Tentative_CH$\neq \phi$ & FOR each $\mathrm{CH} i$ \\
\hline FOR each Tentative_CH $i$ & FOR each neighbor $\mathrm{CH} j$, where hop $(i)>$ hop $(j)$ \\
\hline IF $C H \_P r o b(i) \geq 1$ & and $i \neq j$ \\
\hline Final_CH & $\operatorname{IF}$ dist $(i, j)$ is the minimum among all neighbor \\
\hline Tentatvie_CH $=$ Tentative_CH$-\{i\}$ & $\mathrm{CHs}$ \\
\hline$i$ broadcasts a $\mathrm{CH}$ announcement & $i$ 's parent $\mathrm{CH}$ is $j$ \\
\hline FOR each Tentative_ $\mathrm{CH} j$ & Step VI. Sensed Data Collection \\
\hline IF dist $(i, j) \leq$ ClusterRange $(i)$ & FOR each $C M i$ \\
\hline$C M \leftarrow j$ & transmits sensed data to its parent $\mathrm{CH}$ \\
\hline FOR each $C M j$ & FOR each $\mathrm{CH} i$ \\
\hline$j$ includes $i$ in its $C H$ list & fuses the received data from its cluster members \\
\hline ELSE & transmits fused data to the $B S$ \\
\hline CH_Prob (i) doubles & \\
\hline
\end{tabular}


After the network topology is formed, $C M$ s transmit sensed data to their parent $C H \mathrm{~s}$ in the allocated time frame. Thereafter, $\mathrm{CH}$ s can do further processes like data fusion, then the data will be relayed to the $B S$ through the backbone network.

This step will be executed for $R$ rounds without any adjustment. And the $R$, referred as Stable Interval in following sections, is an application-specific parameter. Once data collection is executed for $R$ rounds, a $C H$ rotation or topology reformation is processed by executing Step II. $\sim$ Step V.

The pseudo code of ACDCR is shown in Table 1. And a sample topology generated by executing ACDCR is shown in Figure 3, in which 200 nodes with random initial energy are dispersed randomly in a $200 * 200$ area. And "* " denotes the BS, " $\times$ " denotes nodes run out of energy, " $\diamond$ ” denotes $C H \mathrm{~s}$, “ $\bigcirc$ " denotes FLCMs, and '+' denotes SLCMs.

\section{Analysis and Simulations}

\subsection{Complexity Analysis}

Lemma 1: ACDCR terminates in $N_{\text {iter }}=O(1)$ iterations.

Proof. The worst case happens when the residual energy level is rather low. Under such situations, the initial value of $C H_{-} \operatorname{Prob}(i)$ will be set to $P_{\min }$, and then doubles after every repetition. Then

$$
2^{N_{\text {iter }}} \times P_{\min } \geq 1
$$

and hence

$$
N_{\text {iter }} \leq\left\lceil\log _{2} \frac{1}{P_{\min }}\right\rceil
$$

Therefore, $N_{\text {iter }} \approx O(1)$. The proof is similar to the proof in [15].

\subsection{Simulation Settings}

Parameters used in the simulation are listed in Table 2. Moreover, to exclude the possibility that the results are outcomes of specific cases, experiments are conducted in two different scenarios and all results are average of 20 experiments. In scenario 1, there are 200 nodes equipped with $0.5 \mathrm{~J}$ batteries, while 400 nodes are deployed with $1 \mathrm{~J}$ initial energy in scenario 2. Besides the above, the widely accepted energy model is assumed in our simulation, the detail of which can be referred in [12, 15-16] and many other articles in this area. And the HEED (AMRP), AMRP refers to Average Minimum Reachability Power, is used as the benchmark algorithm in the simulation. Last of all, all simulation is performed via Matlab 7.1. 
Table 2. Simulation Parameters

\begin{tabular}{|c|c|c|c|}
\hline Parameter & Value & Parameter & Value \\
\hline Number of Nodes & $200 / 400$ & Threshold distance & $\operatorname{sqrt}\left(\varepsilon_{m p} / \varepsilon_{f s}\right)$ \\
\hline Network Grid & $(0,0) \sim(200,200)$ & Intra $_{0}$ & 25 \\
\hline Base Station & $(100,100)$ & Intra $_{\max }$ & 0.15 \\
\hline Initial Energy & $0.5 \mathrm{~J} / 1 \mathrm{~J}$ & $\alpha$ & 0.15 \\
\hline$E_{\text {elec }}$ & $50 \mathrm{~nJ} / \mathrm{bit}$ & Control Packet Size & 25 bytes \\
\hline$\varepsilon_{f s}$ & $10 \mathrm{pJ} / \mathrm{bit} / \mathrm{m} 2$ & Data Packet Size & 100 bytes \\
\hline$\varepsilon_{m p}$ & $0.0013 \mathrm{pJ} / \mathrm{bit} / \mathrm{m}^{4}$ & Stable Interval & 10 \\
\hline$E_{f u s i o n}$ & $5 \mathrm{~nJ} / \mathrm{bit} / \mathrm{signal}$ & & \\
\hline
\end{tabular}

\subsection{Performance Evaluation}

$\checkmark \quad$ Network Lifetime

Table 3. Comparison of lifespan

\begin{tabular}{|c|c|c|c|c|}
\hline $\begin{array}{c}\text { Number of } \\
\text { Nodes }\end{array}$ & $\begin{array}{c}\text { Initial } \\
\text { Energy }\end{array}$ & Algorithm & $\begin{array}{c}\text { Round First } \\
\text { Node Dies }\end{array}$ & $\begin{array}{c}\text { Round Last } \\
\text { Node Dies }\end{array}$ \\
\hline \multirow{2}{*}{200} & \multirow{2}{*}{$0.5 \mathrm{~J}$} & ACDCR & 2422 & 18678 \\
\cline { 3 - 5 } & \multirow{2}{*}{400} & HEED & 1954 & 11987 \\
\cline { 3 - 5 } & $1 \mathrm{~J}$ & ACDCR & 4328 & 37228 \\
\hline
\end{tabular}

Energy conservation has always been the first priority for protocol design in WSNs. The comparison of lifespan between ACDCR and HEED is shown in Table 3. Furthermore, the life curves in the assumed scenarios for both ACDCR and HEED are given in Figure 4 and Figure 5. It can be inferred from the results that ACDCR achieves a longer network lifespan than HEED.

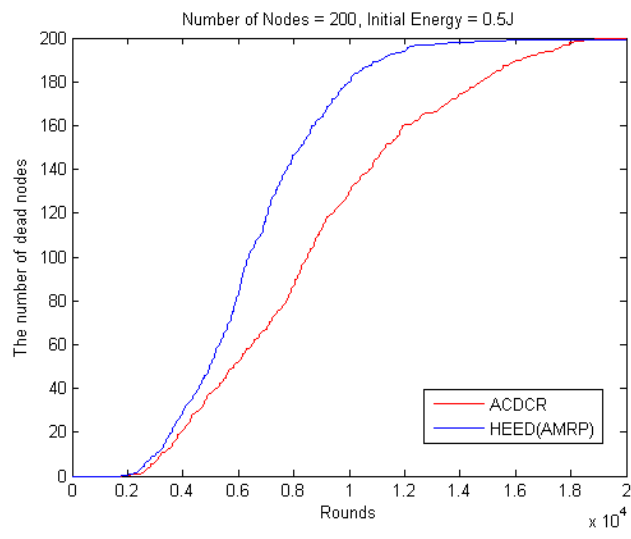

Figure 4. Lifetime Curve under Senario1

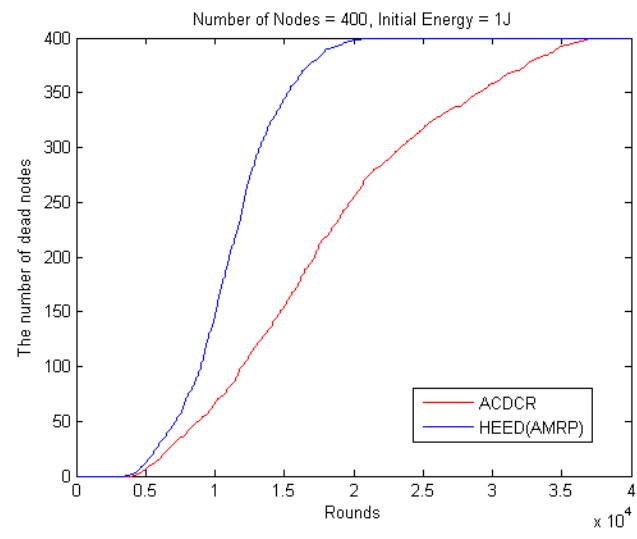

Figure 5. Lifetime Curve under Senario2 


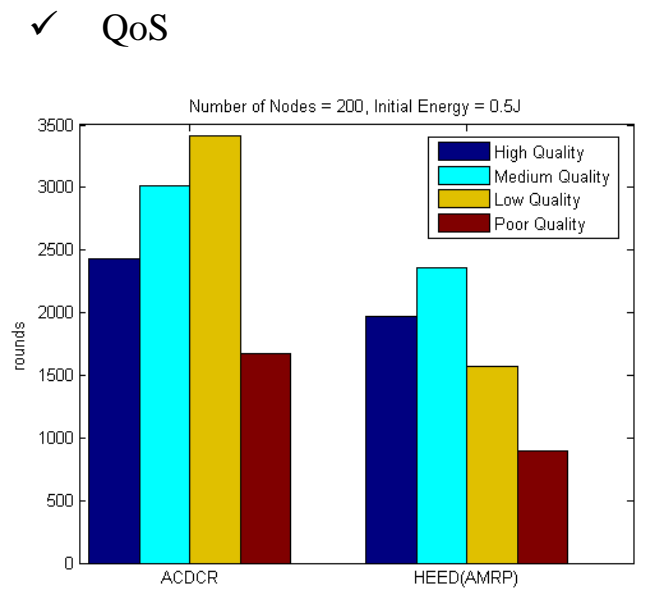

Figure 6. QoS under Senario1

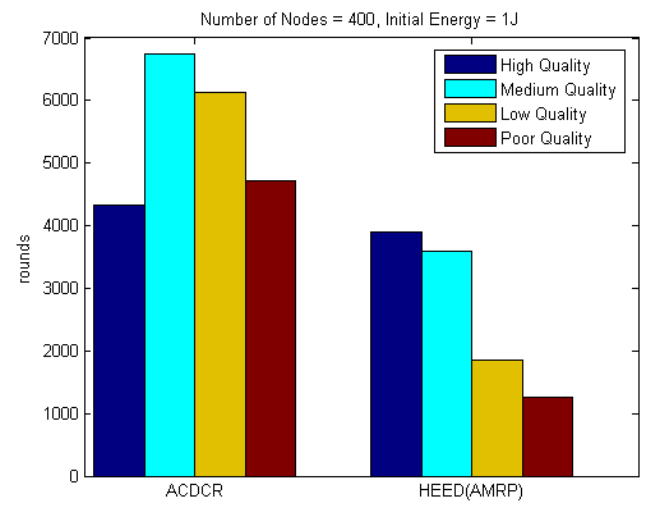

Figure 7. QoS under Senario2

Given the same node distribution, there is no doubt that more active nodes will lead to a better chance for successful event detection. And for simplicity, the problem of coverage overlapping will not be addressed in this study. Based on the above assumption, the number of active nodes can be used as a QoS (Quality of Service) index. Furthermore, the terms for different QoS level are defined as follows.

1. High Quality, all nodes are alive.

2. Medium Quality, node failure begins, but more than 80 percent nodes are alive.

3. Low Quality, less than 80 percent but more than 50 percent nodes are alive.

4. Poor Quality, less than 50 percent but more than 30 percent nodes are alive.

Based on the above definition, the QoS of ACDCR and HEED is compared. The results are illustrated as Figure 6 and Figure 7, and it is safe to say besides longer longevity that ACDCR also has a better QoS as compared to HEED.

\section{$\checkmark \quad$ Percentage of $\mathrm{CH}$}

It is favored for clustering algorithm to have a small amount of $\mathrm{CHs}$, and the percentage of $\mathrm{CH}$ keeps stable in the lifecycle. The comparison illustrated in Figure 8 and Figure 9, implicates that ACDCR has less $\mathrm{CH}$ s and smaller fluctuation in the $\mathrm{CH}$ ratio as compared to HEED.

Such outcomes are gained by the different handling of the 'orphaned' nodes. In HEED, the 'orphaned' nodes, which have no neighbor in their neighborhood area, will become $\mathrm{CH}$. Such setting will inevitably lead to more $\mathrm{CH}$ nodes. Instead of letting 'orphaned' nodes become $\mathrm{CH}$, in ACDCR, they join clusters as SLCMs. 


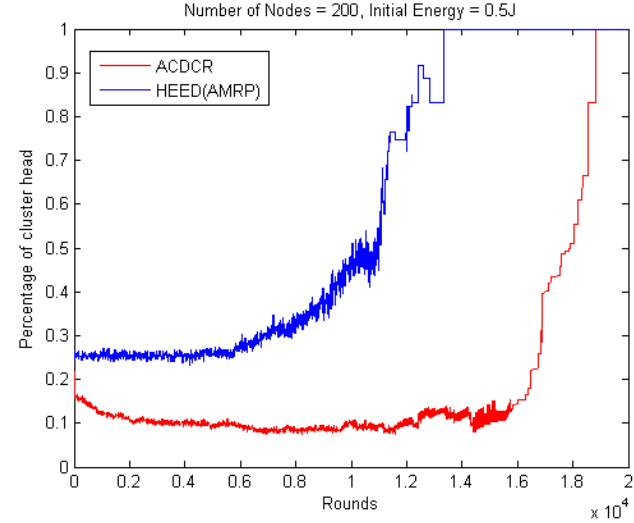

Figure 8. CH Raito under Senario1

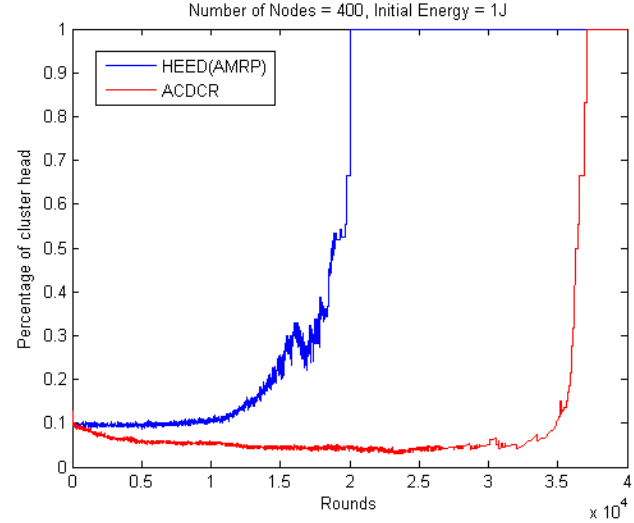

Figure 9. CH Raito under Senario2

\section{Anti-Funneling Effect}

Not unexpectedly, shown by Figure 10 and Figure 11, as nodes die, the network coverage area shrinks while the connectivity is preserved, which is compounded by the cluster radius setting, and allowing the sensor nodes to setup direct link with the $B S$.

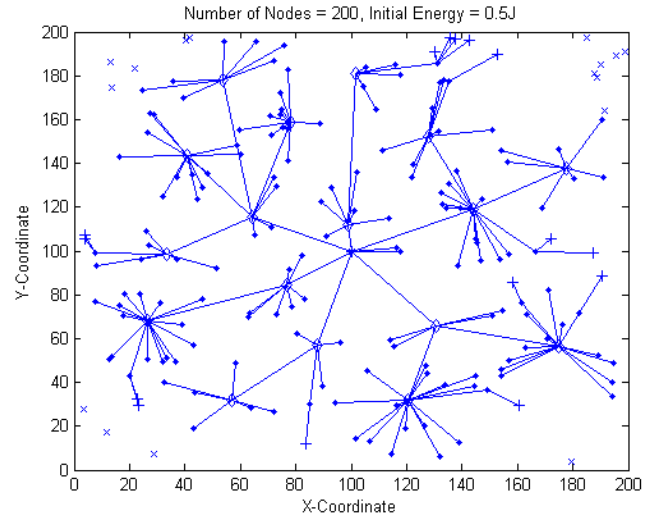

Figure 10. Topology after 4000 rounds

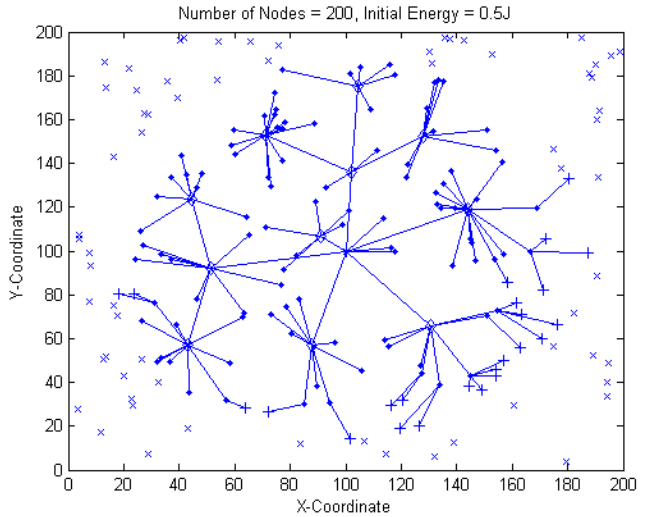

Figure 11. Topology after 8000 rounds

\section{$\checkmark \quad$ Side Effects}

It should be noticed that it is possible that nodes can neither be covered by the $B S$ and $\mathrm{CHs}$, nor $F L C M$ s. However, such situation occurs with very small probability, which is convinced by over 100 experiments (random initial energy level range from 0 to 1,10 rounds each experiment), illustrated in Table 4.

Table 4. Uncovered nodes

\begin{tabular}{|c|c|c|}
\hline $\begin{array}{c}\text { Number of } \\
\text { Nodes }\end{array}$ & $\begin{array}{c}\text { Isolation Occurred } \\
\text { Times }\end{array}$ & $\begin{array}{c}\text { Average Uncovred } \\
\text { Nodes Per Round }\end{array}$ \\
\hline 200 & 3 & 0.3 \\
\hline 400 & 1 & 0.1 \\
\hline
\end{tabular}




\section{Conclusions}

A distributed clustering algorithm called ACDCR is proposed in this paper, which is designed for quasi-stationary networks. And no special assumption, such as location-aware, is made. Moreover, with the strategy for $\mathrm{CH}$ selection and the setting of adaptive cluster radius, it terminates in constant iterations and achieves better performance with ignorable side effect.

For future study, efforts can be made in the node deployment pattern's influence on topology control. Moreover, fault-tolerant mechanisms for inter-cluster communication can be further discussed to achieve better robustness.

\section{Acknowledgements}

This project is supported by the Scientific Research Fund of SiChuan Provincial Education Department under granted NO. 13ZB0088, the Applied Basic Research Programs of Science and Technology Commission Foundation of Sichuan Province under granted NO. 2013JY0064, and the Special Foundation for Young Scientists of Chengdu University of Information Technology.

\section{References}

[1] I. F. Akyildiz, W. Su, Y. Sankarasubramaniam and E. Cayirci, "Wireless Sensor Networks: A Survey", Computer Networks, vol. 38, no. 4, (2002), pp. 393-422.

[2] J. Yick, B. Mukherjee and D. Ghosal, "Wireless sensor network survey", Computer Networks, vol. 52, no. 12, (2008), pp. 2292-330.

[3] T. Bokareva, W. Hu, S. Kanhere, B. Ristic, N. Gordon, T. Bessell, M. Rutten and S. Jha, "Wireless Sensor Networks for Battlefield Surveillance", Proc. Land Warfare Conference 2006, Brisbane, Australia, (2006).

[4] R. Szewczyk, E. Osterweil, J. Polastre, M. Hamilton, A. Mainwaring and D. Estrin, "Habitat Monitoring with Sensor Networks", Communications of the ACM, vol. 47, no. 6, (2004), pp. 34-40.

[5] G. Werner-Allen, K. Lorincz, M. Welsh, O. Marcillo, J. Johnson, M. Ruiz and J. Lees, "Deploying a Wireless Sensor Network on an Active Volcano", IEEE Internet Computing, vol. 10, no. 2, (2006), pp. 18-25.

[6] T. Locher, P. Von Rickenbach and R. Wattenhofer, "Sensor Networks Continue to Puzzle: Selected Open Problems, 9th International Conference on Distributed Computing and Networking, ICDCN 2008, Kolkata, India, (2008), pp. 25-38.

[7] S. Paolo, "Topology Control in Wireless Ad hoc and Sensor Networks", ACM Computing Surveys, vol. 37, no. 2, (2005), pp. 164-194.

[8] R. Banner and A. Orda, "Multi-Objective Topology Control in Wireless Networks", Proc. IEEE INFOCOM 2008, Phoenix, AZ., USA, (2008), pp. 1121-1129.

[9] A. A. Abbasi and M. Younis, "A Survey on Clustering Algorithms for Wireless Sensor Networks", Computer Communications, vol. 30, no. 14-15, (2007), pp. 2826-2841.

[10] J. Sunil and R. Prabhat, "A survey: Topology control for wireless sensor networks", Proceedings of ICSCN 2008 - International Conference on Signal Processing Communications and Networking, Chennai, India, (2008), pp. 422-427.

[11] W. B. Heinzelman, A. P. Chandrakasan and H. Balakrishnan, "An Application-specific Protocol Architecture for Wireless Microsensor Networks", IEEE Transactions on Wireless Communications, vol. 1, no. 4, (2002), pp. 660-670.

[12] W. R. Heinzelman, A. Chandrakasan and H. Balakrishnan, "Energy-efficient Communication Protocol for Wireless Microsensor Networks", Proc. The 33rd Annual Hawaii International Conference on System Sciences, Maui, HI, USA, (2000), p. 10.

[13] K. Xu and M. Gerla, "A Heterogeneous Routing Protocol Based on a New Stable Clustering Scheme", Proc. IEEE Military Communications Conference, Anaheim, CA, (2002), pp. 838-843.

[14] H. Zhang and A. Arora, "GS3: Scalable Self-configuration and Self-healing in Wireless Networks", Computer Networks, vol. 43, no. 4, (2002), pp. 459-480.

[15] O. Younis and S. Fahmy, "HEED: A Hybrid, Energy-efficient, Distributed Clustering Approach for Ad hoc Sensor Networks", IEEE Transactions on Mobile Computing, vol. 3, no. 4, (2004), pp. 366-379.

[16] P. Neamatollahi, H. Taheri, M. Naghibzadeh and M. H. Yaghmaee, "DESC: Distributed Energy Efficient 
Scheme to Cluster Wireless Sensor Networks", Proc. The 9th IFIP TC 6 International Conference 2011, (2011), pp. 234-246.

[17] N. Xu, A. Huang, T.-W. Hou and H.-H. Chen, "Coverage and Connectivity Guaranteed Topology Control Algorithm for Cluster-based Wireless Sensor Networks", Wireless Communications and Mobile Computing, vol. 12, no. 1, (2012), pp. 23-32.

[18] B. Azzedine and M. Anahit, "An Energy-Aware and Fault Tolerant Inter-Cluster Communication based Protocol for Wireless Sensor Networks”, IEEE GLOBECOM 2007 - 2007 IEEE Global Telecommunications Conference, Proceedings, 2007, Washington, DC, United states, (2007), pp. 1164-1168

[19] Y. Adel, Y. M. Youssef, Moustafa and A. Ashok, "Distributed Formation of Overlapping Multi-hop Clusters in Wireless Senor Networks", IEEE GLOBECOM 2006 - 2006 Global Telecommunications Conference, San Francisco, CA, United states, (2006).

[20] P. Ding, J. Holliday and A. Celik, "Distributed Energy Efficient Hierarchical Clustering for Wireless Sensor Networks", Proc. The IEEE International Conference on Distributed Computing in Sensor Systems 2005, Marina Del Rey, CA, (2005), pp. 322-339.

[21] C. Y. Wan, E. E. Shane, T. C. Andrew and C. John, "Siphon: Overload Traffic Management Using Multiradio Virtual Sinks in Sensor Networks", Proceedings of the 3rd International Conference on Embedded Networked Sensor Systems 2005. San Diego, California, USA, (2005), pp. 116-129.

\section{Authors}

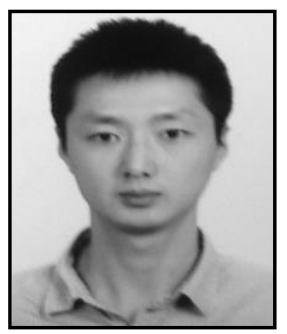

\section{Luqiao Zhang}

He received B.S., M.S. and Ph.D degree in computer science from University of Electronics and Technology of China (UESTC) in 2003, 2006 and 2013, respectively. He is now an associate professor in Chengdu University of Information Technology. His research interests include smart phone and wireless sensor network.

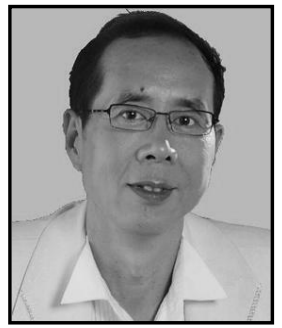

\section{Qingxin Zhu}

He got his B.S. degree on Math in the Math Department of Sichuan Normal University, Chengdu, P.R.C. He was admitted by the Applied Math Department of the Beijing University of Technology in 1982 and got his master's degree there. In 1988, he went to Math and Electrical Engineering Department of the Ottawa University, Canada for his doctor's degree. From May 1993 to March 1995, he did postgraduate research in the Electrical Engineering Department of the Ottawa University and the Computer Department of the Carlton University. Currently he is a professor and doctoral supervisor at the University of Electronic Science and Technology of China. His major research fields are Computer Networks, Bio-informatics, Optimization and Search Theory.

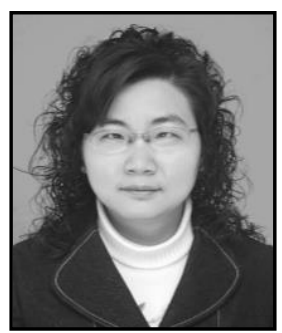

\section{Juan Wang}

He received her M.S. degree of Computer Architecture and Ph.D degree of Information Security from University of Electronics and Technology of China (UESTC) in 2006 and 2010. And being a visiting scholar at University of North Carolina at Charlotte (UNCC) from 2007.9 to 2008.9.Herresearch interests include network security and grid storage. 